Marilana Geimba de Lima 1

Sergio Koifman 2

Ilzia Lins Scapulatempo 3

Maristela Peixoto 1

Silvia Naomi 1

Maristela Curado do Amaral 1

\section{Fatores de risco para câncer de mama em mulheres indígenas Teréna de área rural, Estado do Mato Grosso do Sul, Brasil}

\author{
Risk factors for breast cancer among rural \\ Teréna Indian women in the State of Mato \\ Grosso do Sul, Brazil
}

1 Departamento de Saúde Coletiva, Universidade

Federal do Mato

Grosso do Sul.

Av. Senador Filinto Muller 1480, Campo Grande, MS 79074-460, Brasil.

mgeimba@zaz.com.br

2 Departamento de

Epidemiologia e Métodos

Quantitativos em Saúde,

Escola Nacional

de Saúde Pública,

Fundação Oswaldo Cruz.

Rua Leopoldo Bulhões 1480

Rio de Janeiro, RJ

21041-210, Brasil.

koifman@ensp.fiocruz.br

3 Departamento de

Patologia, Universidade

Federal do Mato

Grosso do Sul.

BR 163, C. P. 549

Campo Grande, MS

79007-900, Brasil.
Abstract In order to evaluate the distribution of sel ected risk factors for breast cancer among Teréna Indian women in the State of Mato Grosso do Sul, Brazil, two samples were intervi ewed, respectively, in 1995 (330 women from ten Indian villages) and 1997 (40 women from the Limão Verde village). Reproductive history, di et characteristics, and family and personal medical history were investigated and body mass index was measured. In the larger sample, mean age at menarche was 12.3 years (30\% at 13 years old or later), $86 \%$ of women reported one or more pregnanci es (42\% reporting 5 or more), $71 \%$ reported three or more pregnancies, mean age at first pregnancy was 18.9 years (3.8\% after 28 years), mean duration of breastfeeding was 84 months, and $70 \%$ reported menopause before age 50 . Di etary pattern in the smaller sample reveal ed a high intake of fruits, vegetables, pasta, and tubers and scarce intake of red meat and chicken; 50 of the women (95\% Cl: 34.1-65.9) presented overweight (BMI 25-29) and 27\% (95\% Cl: 15.1-44.1) obesity (BMI >29).

Key words Breast Neoplasms; South American Indians; Risk Factors

Resumo Com o objetivo de se avaliar a distribuição de fatores de risco em mulheres indígenas Teréna no Estado do Mato Grosso do Sul, duas amostras destas foram entrevistadas, respectivamente em 1995 (330 mul heres vol untárias de dez al dei as teréna de área rural) e em 1997 (quarenta mulheres da Al deia de Limão Verde). Os padrões de vida reprodutiva, di eta, antecedentes patológi cos pessoai s efamiliares foram levantados, bem como a determi nação do índice de massa corporal (IMC). Na maior amostra, observou-se quea idade média à menarca foi de 12,3 anos ( $30 \%$ das mulheres referindo-a aos 13 anos ou mais), $86 \%$ das mul heres com antecedentes de uma ou mais gestações (42\% com cinco ou mais), $71 \%$ com antecedentes de três ou mais gestações, idade média à primeira gestação de 18,9 anos (3.8\% com 28 anos ou mais), aleitamento materno com duração médi a de 84 meses, e 70\% das mulheres menopausadas antes dos cinqüenta anos de idade. O padrão referi do de di eta na menor amostra revela el evado consumo de frutas, legumes, raízes e macarrão, com ingestão escassa de carne vermel ha e frango, observando-se que 50\% (IC 95\%: 34.1-65.9) das mulheres apresentaram sobrepeso (IMC 25-29) e $27 \%$ (IC 95\%: 15.1-44.1) obesidade (IMC >29).

Palavras-chave Neoplasias Mamárias; Índios Sul-Americanos; Fatores de Risco 


\section{Introdução}

A elevação da incidência do câncer de mama no Brasil éum fato marcante no quadro de saúde pública de sua população, em que esta neoplasia já ocupa a primeira causa de câncer em mulheres no país com um todo (MS, 2000). Na Região Centro-oeste, a incidência acumulada do câncer de mama em Goiânia, Goiás, determinada com base nos dados coletados pelo Registro de Câncer de Base Populacional desta cidade no início da década de 90 (Parkin et al., 1992), foi estimada em $6,5 \%$, ou seja, a existência de um risco previsto de desenvolvimento de um caso de câncer de mama em cada 16 muIheres nascidas neste período e que venham a viver até os oitenta anos de idade. Cifras ainda mais elevadas, da ordem de $8 \%$ de risco, foram observadas na Cidade de São Paulo, ou seja, de um caso de câncer em cada 12 mulheres ao longo da vida (Koifman \& Koifman, 1997).

Esta elevação da incidência do câncer de mama tem sido constatada em diversos países industrializados ou em fase de industrialização, sendo objeto de preocupação dada a incapacidade tecnológica atual de prevenção da doença. Desta maneira, as medidas de sua detecção precoce consistem nos instrumentos atualmente disponíveis de modificação da história natural do câncer de mama, e assim, de controle de sua letalidade, ampliação da sobrevida e da qualidade de vida das mulheres afetadas (Koifman \& Koifman, 1999).

À luz dos conhecimentos atuais (Kelsey \& Horn-Ross, 1993), a estimulação ao processo de divisão celular das células mamárias, regida pelos ciclos ovulatórios ao longo da vida reprodutiva da mulher, poderia conduzir ao surgimento potencial de células mal-formadas, suscetíveis à ação de substâncias carcinogênicas. Desta maneira, aqueles eventos pautados pela ampliação da estimulação hormonal estrogênica, potencializada pela progesterona durante a fase lútea do ciclo, acarretariam um estímulo ao processo de divisão celular das células da mama. A precocidade da menarca, a existência de menopausa em fases tardias da vida reprodutiva, a reduzida paridade, entre outros, constituem eventos marcadores de um processo de estimulação hormonal continuada e que induzem a proliferação das células mamárias (Hardy et al., 1993).

Por outro lado, o desenvolvimento da primeira gravidez parece representar um importante evento no processo de maturação das células da mama, tornando-as potencialmente mais protegi das em relação à ação de substâncias cancerígenas. Desta forma, a nuliparidade, bem como o retardo na primiparidade, consistiriam em fatores de risco para o desenvolvimento do câncer de mama. (Apter, 1996; Canty, 1997; Tavani et al., 1999). A atenção dos pesquisadores tem sido freqüentemente direcionada para a análise da distribuição do câncer de mama e de outras neoplasias em diferentes populações, com o objetivo de estabelecer padrões diferenciais de sua ocorrência e, por conseguinte, identificar possíveis explicações para as diferenças encontradas.

$A$ atenção de um dos autores do presente trabalho (M. G. L.) foi despertada para a observação na prática clínica da mastologia quanto à aparente inexistência de casos de câncer de mama identificados na demanda de mulheres teréna e familiares - observação clínica sem confirmação epidemiológica - nos serviços públicos de saúde ginecológica em Mato Grosso do Sul, paral elamente à constatação de que o mesmo fenômeno não se verificava em relação a casos de câncer em outras local izações anatômicas.

Desta maneira, foi desenvolvido um projeto de investigação para determinar o padrão de distribuição de fatores de risco reprodutivo e outros associados com aquela neoplasia em mulheres teréna, com o intuito de sua posterior comparação com o padrão epidemiológico similar descrito na população geral brasileira. Este trabalho apresenta os resultados preliminares de um inquérito realizado para atender aquele objetivo.

\section{Metodologia}

A população Teréna residente no Estado do Mato Grosso do Sul é constituída de aproximadamente vinte mil pessoas, representando $38 \%$ da comunidade indígena daquele Estado e 8,5\% de toda a população indígena no Brasil (CEDI/ PETI, 1990; Mangolin, 1993). Sua origem está relacionada aos povos Aruák, subgrupo Guaná ou Chaná, oriundos das planícies da Colômbia, Venezuela, Equador e Chaco paraguaio, tendo desenvolvido importante papel na formação histórica da Região Centro-oeste como produtores de bens para os primeiros colonizadores. Após a Guerra do Paraguai, para a qual foram recrutados, foram criadas as reservas indígenas, tendo retornado a suas aldeias ou ao trabal ho nas fazendas (Oliveira, 1976). Desde então, tem se dedicado à agricultura de subsistência por intermédio das culturas de arroz, feijão, milho e mandioca, que constituem a base de sua alimentação (Altonfelder, 1949; Mangolin, 1993). As mulheres dedicam-se à realiza- 
ção de trabalhos domésticos, fiação e confecção de cerâmica, enquanto os homens têm suas principais atividades voltadas para a caça, pesca e agricultura (Oberg, 1990). Graças a estas atividades, tem conseguido manter um modo de vida relativamente autônomo, dispondo de terras para moradia e plantio, bem como assegurando sua não-assimilação e a preservação de sua identidade cultural (Oliveira, 1976).

Um inquérito para traçar o perfil epidemiológico reprodutivo em mulheres Teréna foi realizado em 1995, em nove aldeias localizadas no Município de Aquidauana (Taunay, Morrinho, Córrego Seco, Imbiruçu, I pegue, Água Branca, Lagoinha, Limão Verde e Bananal) e uma aldeia (Aldeinha) no Município de Anastácio, Mato Grosso do Sul. Segundo a Fundação Nacional do Índio (FUNAI), essas contavam, em 1993, com uma população total de 3.569 índios nas aldeias localizadas no Município de Aquidauana e 328 nas de Anastácio.

Com base em um trabalho de informação prévio realizado por agentes comunitários das aldeias, 330 mulheres se apresentaram como voluntárias para participação, sendo realizada entrevista. Durante esta, foi empregado um instrumento padronizado (questionário) de coleta de dados sobre a idade, estado civil, variáveis reprodutivas, antecedentes pessoais e familiares de câncer e tabagismo. Após a entrevista, foi realizado exame físico da região mamária e coleta de material citopatológico de colo uterino (resultados não apresentados neste trabalho). Ambas as atividades (entrevista e exame) foram desenvolvidas na presença de auxiliar de enfermagem ou agente comunitária indígena para auxílio com o idioma teréna.

Posteriormente, em 1997, uma segunda amostra de quarenta mulheres ( $15 \%$ da população feminina com idade igual ou superior a vinte anos) da aldeia de Limão Verde, Município de Aquidauana, foi entrevistada a respeito de hábitos alimentares e prática de atividade física, bem como tendo realizado exame clínico ginecológico. Nesta amostra determinou-se o índice de massa corporal (IMC) valendo-se da razão entre as medidas de peso e quadrado da al tura para cada entrevistada (Anjos, 1992) (Tabela 1).

\section{Resultados}

Na análise da amostra de mulheres das dez aldeias Teréna participantes deste estudo, 80\% referiram situação matrimonial estável, sendo $15,3 \%$ solteiras, 3,9\% viúvas e apenas três mulheres referiram ser separadas/ divorciadas $(0,9 \%)$.
Cerca de $86 \%$ tiveram pelo menos uma gestação, com uma média de cinco gestações, tendo $42 \%$ referido cinco ou mais gestações ao longo da vida (Tabela 2). Entre as que engravidaram (283), 98,6\% evoluíram a gestação a termo, sendo observada uma média de 4,6 filhos por mulher, tendo apenas $1,4 \%$ referido a prática de abortamento.

Entre as entrevistadas que gestaram, a idade média à primi paridade foi 18,9 anos, tendo ocorrido durante a puberdade em $5,3 \%$ destas, em 34\% durante a adolescência e em 3,8\% após os 28 anos de idade (Tabela 2).

A idade média à menarca foi 12,3 anos, observando-se que em $2,8 \%$ das mulheres esta ocorreu antes dos 11 anos de idade, e em 30\% a partir dos 13 anos de idade (Tabela 2). Das 51 mulheres que haviam alcançado a menopausa, 36 (70\%) o fizeram antes dos cinqüenta anos de idade.

A utilização de métodos anticoncepcionais hormonais foi referida por 13 entrevistadas (4\%), tendo $64(19,4 \%)$ referido anticoncepção cirúrgica, e 77\% a não-utilização de qualquer método. $\mathrm{O}$ tabagismo foi referido por apenas $7 \%$ das mulheres. Relatos de antecedentes familiares de câncer foram mencionados por 29 entrevistadas (8,8\%), sendo todos de local ização extramamária ( 14 casos de câncer uterino e 15 em outras localizações). Antecedentes pessoais de câncer entre as entrevistadas foram mencionados por sete mulheres $(2,1 \%)$, sendo quatro casos de câncer uterino e um de tumor genital.

$\mathrm{Na}$ análise da amostra de quarenta mulheres da aldeia de Limão Verde, realizada em 1997,

Tabela 1

Distribuição etária das amostras de voluntárias. Aldeias Teréna, municípios de Aquidauana e Anastácio, Mato Grosso do Sul, 1995 e 1997.

\begin{tabular}{lrrrr}
\hline \multirow{2}{*}{ Idade (anos) } & \multicolumn{2}{c}{$\begin{array}{c}\text { Amostra* } \\
\text { n }\end{array}$} & $n$ & \multicolumn{2}{c}{$\begin{array}{c}\text { Aldeia Limão Verde } \\
n\end{array}$} \\
\hline$<20$ & 48 & 14,6 & - & - \\
$20-29$ & 101 & 30,6 & 13 & 32,5 \\
$30-39$ & 73 & 22,1 & 13 & 32,5 \\
$40-49$ & 33 & 10,0 & 5 & 12,5 \\
$50-59$ & 35 & 10,6 & 3 & 7,5 \\
$60-69$ & 17 & 5,2 & 5 & 12,5 \\
$>69$ & 15 & 4,6 & 1 & 2,5 \\
Ignorada & 8 & 2,4 & - & - \\
Total & 330 & 100,0 & 40 & 100,0 \\
\hline
\end{tabular}

* amostra de voluntárias das aldeias Taunay, Morrinho, Córrego Seco, Imbiruçu, Ipegue, Água Branca, Lagoinha, Limão Verde, Bananal (Município de Aquidauna, Mato Grosso do Sul) e Aldeinha (Município de Anastácio, Mato Grosso do Sul). 


\begin{tabular}{|c|c|c|}
\hline \multicolumn{3}{|c|}{$\begin{array}{l}\text { Distribuição de freqüências de variáveis } \\
\text { reprodutivas selecionadas. Amostra de mulheres } \\
\text { Teréna, municípios de Aquidauana e Anastácio, } \\
\text { Mato Grosso do Sul, } 1995 \text {. }\end{array}$} \\
\hline Variável reprodutiva & $\mathrm{n}$ & $\%$ \\
\hline \multicolumn{3}{|l|}{ Número de gestações } \\
\hline 0 & 47 & 14,2 \\
\hline $1-2$ & 73 & 22,1 \\
\hline $3-4$ & 73 & 22,1 \\
\hline $5-7$ & 78 & 23,7 \\
\hline $8-10$ & 38 & 11,5 \\
\hline 11 ou mais & 21 & 6,4 \\
\hline Total & 330 & 100,0 \\
\hline \multicolumn{3}{|l|}{ Paridade } \\
\hline $1-2$ & 80 & 28,6 \\
\hline $3-4$ & 75 & 26,9 \\
\hline $5-7$ & 74 & 26,5 \\
\hline $8-10$ & 37 & 13,3 \\
\hline 11 ou mais & 13 & 4,7 \\
\hline Total & 279 & 100,0 \\
\hline \multicolumn{3}{|l|}{ Idade da 1a gestação } \\
\hline $12-14$ & 14 & 5,2 \\
\hline $15-17$ & 89 & 33,2 \\
\hline $18-24$ & 145 & 54,2 \\
\hline $25-27$ & 10 & 3,7 \\
\hline 28 ou mais & 10 & 3,7 \\
\hline Total & 268 & 100,0 \\
\hline \multicolumn{3}{|l|}{ Idade à menarca } \\
\hline $8-10$ & 9 & 2,9 \\
\hline $11-12$ & 206 & 66,7 \\
\hline $13-14$ & 75 & 24,3 \\
\hline $15-17$ & 15 & 4,9 \\
\hline 18 e mais & 4 & 1,3 \\
\hline Total & 309 & 100,0 \\
\hline
\end{tabular}

resultados similares foram observados em relação ao número de gestações (média de 3,8 gestações, desvio-padrão de 2,6), paridade (média de 3,72 filhos, desvio-padrão de 2,6), idade materna à primeira gestação (média de 20,4 anos, desvio-padrão de 5,6), idade à menarca (média de 12,1 anos, desvio-padrão de 0,9). A duração média do aleitamento materno ao longo da vida foi de 84 meses, a prevalência de tabagismo de $1,5 \%$, tendo sido referidos antecedentes familiares de câncer em parentes de primeiro grau em $\mathbf{2 5 \%}$ das entrevistadas, sendo estes também de localização extramamária (cinco casos de câncer uterino, quatro de boca e uma leucemia).
Somente duas mulheres referiram uso de anticoncepcional hormonal (com duração de 3 e 4 anos, respectivamente), enquanto dezesseis (40\%) referiram ter sido submetidas à esterilização cirúrgica. Não houve relatos de consumo de bebidas alcoólicas nem da prática de esportes ou atividades físicas regulares neste grupo. O padrão de distribuição do IMC determinado nesta amostra foi o seguinte: IMC < 19: uma mulher (2,5\%, IC 95\%: 0,1-14,7); IMC 19-24: oito mulheres (20,0\%, IC 95\%: 9,6-36,1); IMC 2529 (sobrepeso): vinte mulheres (50\%, IC 95\%: 34,1-65,9); e IMC > 29 (obesidade): 11 Mulheres (27,5\%, IC 95\%: 15,1-44,1).

A Tabela 3 apresenta distribuição de freqüências do padrão de consumo alimentar durante as refeições na amostra estudada, evidenciando-se elevada prevalência de consumo de carboidratos em todas as refeições na amostra estudada. Nos intervalos entre refeições, foram obtidos relatos sobre o consumo usual de frutas de estação coletadas na própria aldeia, tais como caju, mamão, banana, laranja e manga.

\section{Discussão}

Muito pouco se conhece sobre as condições da saúde da mulher entre os povos indígenas situados no Brasil, incluindo aspectos relativos à epidemiologia de certas neoplasias. Este trabaIho teve como ponto de partida a constatação, baseada em evidências clínicas, mas que carecia de suporte epidemiológico, quanto à nãoobservação de casos de câncer de mama em mulheres Teréna. Esse fato, que poderia também ser devido ao reduzido contingente populacional analisado, estimulou a organização de um inquérito que permitisse analisar a distribuição de fatores de risco associados a tal neoplasia neste grupo populacional.

Este estudo não se baseia em uma amostra de caráter aleatório. Há, portanto, a possibilidade de ocorrência de possíveis tendenciosidades quanto à percepção de seu estado de saúde e condicionantes, acarretada pelo fato dos indivíduos participantes terem se apresentado voluntariamente. A opção de seleção das participantes decorreu de dificuldades inerentes à realização de um estudo empregando dois idiomas (Português e Teréna), bem como em função da esperada resistência cultural relacionada à sua implementação numa população indígena com antecedentes de relações complexas com a sociedade nacional e seus representantes. Outro fator potencialmente importante na introdução de tendenciosidades diz respeito à necessidade de recordação de fatos ocorri- 
dos há décadas passadas, como a idade da menarca, sobretudo nas mulheres mais idosas.

A inexistência de relatos de casos de câncer de mama na população de mulheres Teréna de origem rural que geraram as amostras analisadas neste trabal ho não deve apontar para generalizações, uma vez que a ausência de casos desta neoplasia poderia resultar do acaso. Por outro lado, acredita-se ser pouco provável a existência de subnotificação de casos de câncer de mama nestas comunidades, em decorrência de dificuldades de acesso aos serviços de saúde, visto que foram observados relatos afirmativos de antecedentes de câncer em outras localizações anatômicas.

Crê-se que a importância de se descrever o padrão de distribuição dos fatores de risco para o câncer de mama em diferentes grupos populacionais pode vir a apontar algumas evidências de interesse para a compreensão dos mecanismos multicausais envolvidos na história natural desta doença. Desta maneira, os padrões de distribuição de variáveis reprodutivas e da dieta emergentes desta análise apontam para certas considerações importantes a serem destacadas.

Em primeiro lugar, verifica-se que o padrão de variáveis reprodutivas observado (Tabela 2 ) caracteriza um perfil que poderia ser genericamente apontado como protetor (diminuição de risco) em relação ao desenvolvimento do câncer de mama: $2,8 \%$ de mulheres com menarca precoce (menos de 11 anos), menopausa com idade média de 47 anos, elevada distribuição de gestações a termo (63\% com três ou mais gestações), aleitamento materno prolongado (sete anos em média), reduzida nuliparidade (14\%), elevada prevalência de primiparidade entre 18 e 27 anos de idade (59\%), e prevalência reduzida da última gestação em idade reprodutiva avançada ( $23 \%$ com idade superior a 31 anos de idade).

Inúmeros relatos etnográficos e demográficos sobre os povos indígenas situados no Brasil apontam para o início das práticas reprodutivas em mulheres já na adolescência, não raro por volta dos 13-14 anos, e que se estendem, com freqüência, até os 40-45 anos (Adams \& Price, 1994; Early \& Peters, 1990; Flowers, 1994). Outro aspecto evidenciado nesses estudos diz respeito à longa extensão do período de aleitamento observado em diversos grupos. Early \& Peters (1990) estimaram que as mulheres Yanomámi, devido à alta fecundidade e à prática freqüente de aleitamento, passam, em média, 92\% de seu período reprodutivo ou grávidas, ou amamentando. Deduz-se, portanto, que as mulheres Yanomámi devem experimentar ao
Tabela 3

Relato de consumo alimentar usual. Amostra de mulheres Teréna*, aldeia de Limão Verde, Município de Aquidauana, Mato Grosso do Sul, Brasil, 1997.

\begin{tabular}{|c|c|c|c|c|c|c|}
\hline \multirow[t]{2}{*}{ Item } & \multicolumn{2}{|c|}{ Desjejum } & \multicolumn{2}{|c|}{ Almoço } & \multicolumn{2}{|c|}{ Jantar } \\
\hline & $\mathrm{n}$ & $\%$ & $n$ & $\%$ & $n$ & $\%$ \\
\hline Mandioca & 13 & 32,5 & 30 & 75,0 & 12 & 30,0 \\
\hline Arroz & 14 & 35,0 & 40 & 100,0 & 14 & 35,0 \\
\hline Feijão & 3 & 7,5 & 40 & 100,0 & 16 & 40,0 \\
\hline Farofa & 1 & 2,5 & 6 & 15,0 & - & - \\
\hline Milho & - & - & 12 & 30,0 & 4 & 10,0 \\
\hline Tomate & - & - & 6 & 15,0 & - & - \\
\hline Batata & - & - & 3 & 7,5 & 8 & 20,0 \\
\hline Frango & - & - & 12 & 80,0 & 2 & 5,0 \\
\hline Carne vermelha & - & - & 20 & 50,0 & 2 & 5,0 \\
\hline Abóbora & - & - & 6 & 15,0 & - & - \\
\hline Macarrão & - & - & 18 & 45,0 & 32 & 80,0 \\
\hline Extrato de tomate & - & - & 6 & 15,0 & 20 & 50,0 \\
\hline Café & 20 & 50,0 & - & - & - & - \\
\hline Açúcar & 13 & 32,5 & - & - & - & - \\
\hline Pão & 27 & 67,5 & - & - & - & - \\
\hline Chá-mate & 25 & 62,5 & - & - & - & - \\
\hline Leite & 9 & 22,5 & - & - & - & - \\
\hline Fruta & 2 & 5,0 & - & - & - & - \\
\hline Bolo & 5 & 12,5 & - & - & - & - \\
\hline O vo & 3 & 7,5 & - & - & - & - \\
\hline Margarina & 1 & 2,5 & - & - & - & - \\
\hline
\end{tabular}

$* n=40$ mulheres

Iongo de suas vidas uma menor quantidade de ciclos ovulatórios, algo que talvez possa ser comum entre as mulheres de muitos outros gruposindígenas.

Embora o papel do emprego de anovulatórios e a prática de abortamento na história natural do câncer de mama seja controverso (Brinton et al., 1997; Canty, 1997), a prevalência de ambos, nas amostras de mulheres Teréna analisadas, foi reduzida.

Em seu conjunto, os resultados do presente estudo indicam para um padrão reduzido de exposição hormonal endógena e exógena nas mulheres Teréna ao longo de sua vida reprodutiva, em decorrência de uma menor freqüência do número de ciclos ovulatórios. Assim, tanto estas apresentam um relativo retardo médio na idade de início de sua vida reprodutiva, quanto precocidade em seu término. Vida reprodutiva que é freqüentemente intercalada por numerosas gestações, seguidas de aleitamento prolongado, eventos estes acompanhados de supressão dos ciclos ovulatórios. Logo, uma muIher Teréna não apenas parece usualmente adquirir o efeito protetor do processo de pleno 
desenvolvimento das células mamárias em decorrência da primeira gestação a termo em idade reprodutiva precoce, como também tende a apresentar redução no número total de ciclos ovulatórios - estimuladores da mitose das células mamárias - ao longo de sua vida.

Portanto, poder-se-ia supor que as mulheres Teréna estariam, em média, menos propensas à formação de células mamárias atípicas, passíveis de interagirem com agentes cancerígenos no ambiente, fato este decorrente sobremodo de sua plena maturação advinda de gestações a termo em idade jovem, bem como de uma menor ocorrência de estímulos contínuos ao processo de divisão celular na mama.

O segundo aspecto de destaque para ser avaliado diz respeito ao perfil alimentar observado na amostra analisada de mulheres da aldeia de Limão Verde. Ainda que a compreensão sobre o papel da dieta no desenvolvimento do câncer de mama apresente controvérsias (Howe et al., 1990; Kohmeier \& Mendez, 1997), existem evidências de que gordura animal e consumo de bebidas alcoólicas (Haile et al., 1996; Kohlmeier \& Mendez, 1997; Nagao et al., 1994; Smith et al., 1994) atuem como fatores de risco, enquanto o consumo de frutas e vegetais ricos em fibras e antioxidantes desempenhem papel de fatores de proteção para o câncer de mama (Freudenheim et al., 1996; Pawlega, 1992; Rose, 1990; Yuan et al., 1995). Em relação ao consumo de gordura animal, suspeita-se de uma possível ação carcinogênica da dieta rica neste item durante a infância, especialmente na adolescência, quando se acentua o processo de divisão celular na mama. Portanto, acredita-se que a mesma possa apresentar uma contribuição importante para o câncer de mama e para outras neoplasias endócrinas (próstata e ovário) em que tais relações estão melhor estabelecidas (Gammon \& John, 1993).

Uma segunda visita ao campo revelou que as informações, inicialmente fornecidas pelas participantes quanto ao consumo de carne vermelha e frango (Tabela 3) sugeriam ter sido artificialmente infladas, em decorrência da concepção do que consideravam ser habitual no padrão alimentar da população não indígena. Neste sentido, o padrão alimentar emergente, tomando-se por base tais correções, é o de uma dieta rica em carboidratos, frutas e legumes, com baixo consumo de gordura animal não comercializada nas al deias estudadas, bem como a quase inexistência do consumo de bebidas alcoólicas. Este perfil alimentar, associado à ausência de uma prática regular de atividades físicas, parece refletir-se na elevada prevalência de obesidade observada na amostra da aldeia de Limão Verde, sendo ambos fatores de risco para o câncer de mama (Magnuson et al., 1998; Swanson et al., 1996; Ziegler, 1997). As elevadas prevalências de sobrepeso e obesidade entre as mulheres Teréna, da ordem de, respectivamente, 50\%(34,1-65,9) e $27 \%(15,1-44,1)$, indicando portanto um excesso ponderal em mais de três quartos das mulheres analisadas, parecem resultar de um padrão de vida intensamente sedentário, desenvolvido numa comunidade ainda parcamente inserida enquanto força de trabalho na sociedade de mercado brasileira, e vivendo com os frutos obtidos de uma economia de subsistência, com elevado consumo alimentar de carboidratos.

Em resumo, o padrão de distribuição de variáveis reprodutivas e da dieta identificado na amostra analisada de mulheres Teréna poderia ser categorizado, em seu conjunto, como de baixo risco para o desenvolvimento de câncer de mama, exceção feita aos níveis de sedentarismo, sobrepeso e obesidade observados. Futuramente, pretende-se avaliar a persistência ou modificação destes padrões em mulheres desta comunidade que migraram para centros urbanos como Campo Grande, Mato Grosso do Sul, e se estas mudanças, porventura existentes, foram também acompanhadas de modificações no padrão de distribuição dos fatores de risco associados ao câncer de mama.

$\mathrm{Na}$ atualidade, pouco se conhece sobre as condições da saúde entre os povos indígenas no Brasil, e particularmente, os aspectos relativos à epidemiologia de certas neoplasias. Neste sentido, o presente trabalho é um dos poucos estudos brasileiros a se deter na análise da distribuição de certos fatores de risco para o câncer em uma comunidade indígena no país. Assim, é de grande importância que se amplie esta área de investigação, documentando as características epidemiológicas da distribuição de câncer nas populações indígenas brasileiras. Este esforço permitirá a criação de mecanismos de intervenção nas condições de vida e de atenção à saúde que venham a possibilitar não apenas a documentação das possíveis razões para sua ocorrência (modificações nos hábitos de vida e exposições ambientais a agentes cancerígenos diversos), mas também viabilizar a implantação de serviços de atenção continuada - como a realização do exame citológi co de Papanicolau - capazes de interferir eficientemente na evolução de alguns tipos de câncer. 


\section{Conclusões}

A análise da distribuição de variáveis reprodutivas por intermédio de inquérito de saúde realizado em duas amostras de mulheres indígenas Teréna de área rural no Estado de $M$ ato Grosso do Sul revelou a ocorrência de $2,8 \%$ de menarca antes dos 11 anos de idade, $71 \%$ das mulheres com antecedentes de três ou mais gestações (55\% com cinco ou mais), idade média à primeira gestação de 19 anos, e 70\% com menopausa antes dos cinqüenta anos de idade. Em relação ao padrão de adequação nutricional, foi observada prevalência de sobrepeso (IMC 25-29) e de obesidade, respectivamente, de 50\% (IC 95\%: 34,1-65,9) e 27\% (IC 95\%: 15,1$44,1)$.

\section{Agradecimentos}

Os autores agradecem à Fundação Nacional do Índio e às Secretarias Municipais de Saúde de Aquidauana e Anastácio, Mato Grosso do Sul, o apoio técnico-financeiro prestado para o desenvolvimento desta investigação, bem como manifestam sua gratidão ao Prof. Ricardo Ventura pelas valiosas sugestões e revisão crítica do texto.

\section{Referências}

ADAMS, K. \& PRICE, D., 1994. The Demography of Small-Scale Societies: Case Studies from Lowland South America. South American Indian Studies 4. Bennington: Bennington College.

ALTENFELDER, S. F., 1949. Mudança cultural dos Terena. Revista do Museu Paulista, Nova Série, 3: 271-379.

ANJOS, L. A., 1992. Índice de Massa Corporal (massa corporal/ estatura2) como indicador do estado nutricional de adultos: Revisão da literatura. Revista de Saúde Pública, 26:431-436.

APTER, D., 1996. Hormonal events during female puberty in relation to breast cancer risk. European Journal of Cancer Prevention, 5:476-482.

BRINTON, L. A.; GAMMON, M. D.; MALONE, K. E.; SCHOENBERG, J. B. \& DALING, J. R., 1997. Modification of oral contraceptive relationships on breast cancer risk by selected factors among younger women. Contraception, 55:197-203.

CANTY, L., 1997. Breast cancer risk: Protective effect of an early first full-term pregnancy versus increased risk of induced abortion. Oncology Nursing Forum, 24:1025-1031.

CEDI (Centro Ecumênico de Documentação e Informação)/ PETI (projeto estudo sobre Terras Indígenas no Brasil), 1990. Terras Indígenas do Brasil. São Paulo: Publicações Cedi. 
EARLY, J. D. \& PETER, J. F., 1990. The Population Dynamics of the Mucajai Yanomama. San Diego: Academic Press.

FLOWERS, N., 1994. Crise e recuperação demográfica: Os Xavánte de Pimentel Barbosa. In: Saúdee Povos Indígenas (R. V. Santos \& C. E. A. Coimbra Jr., org.), pp. 213-242, Rio de Janeiro: Editora Fiocruz.

FREUDENHEIM, J. L.; MARSHALL, J. L. R \&VENA, J. E., 1996. Premenopausal breast cancer risk and intake of vegetables, fruits and related nutrients. Journal of the National Cancer Institute, 88:340-348.

GAM MON, M. \&JOHN, E., 1993. Recent etiologic hypothesis concerning breast cancer. Epidemi ologic Reviews, 15:163-168.

HAILE, R. W.; WITTE, J. S. \& URSIN, G., 1996. A casecontrol study of reproductive variables, alcohol, and smoking in premenopausal bilateral breast cancer. Breast Cancer Research and Treatment, 37: 49-56.

HARDY, E. E.; PINOTTI, J. A.; OSIS, M. J. D. \& FAÚNDES, A., 1993. Variáveis reprodutivas e risco para câncer de mama: Estudo caso-controle desenvolvido no Brasil. Boletín dela Oficina Sanitaria Panamericana, 115:93-102.

HOWE, G. R.; HIROHATA, T.; HISLOP, T. J.; ISCOVICH, J. M.; YUAN, J. M. \& KATSOUYANNIK, K., 1990. Dietary factors and risk of breast cancer: Combined analysis of 12 case-control studies. Journal of the National Cancer Institute, 82:561-569.

KELSEY, J. L. \& HORN-ROSS, P. L., 1993. Breast cancer: Magnitude of the problem and descriptive epidemiology. Epidemi ologic Reviews, 15:7-16.

KOHMEIER, L. \& MENDEZ, M., 1997. Controversies surrounding diet and breast cancer. Proceedings of the Nutrition Society, 56(1B):369-382.

KOIFMAN, S. \& KOIFMAN, R. J., 1997. Epidemiological trends of female breast cancer in Brazil. Breast Cancer Research and Treatment, 49:41.

KOIFMAN, S. \& KOIFMAN, R. J., 1999. Incidência e mortalidade por câncer no Brasil. In: Questões da Saúde Reprodutiva (K. Giffin \& S. H. Costa, org.), pp. 227-251, Rio de Janeiro: Editora Fiocruz.

MAGNUSSON, C.; BARON, J.; PERSON, I.; WOLK, A.; BERGSTROM, R.; TRICHOPOULOS, D. \& ADAMI, H. O., 1998. Body size in different periods of life and breast cancer risk in post-menopausal women. International Journal of Cancer, 76:29-34.
MANGOLIN, O., 1993. Povos Indígenas no Mato Grosso do Sul: Viveremos por mais 500 Anos. Campo Grande: Conselho Indigenista Missionário.

MS (Ministério da Saúde), 2000. Estimativas 2000. Brasília: Instituto Nacional do Câncer, Ministério da Saúde.

NAGAO, M.; USHIJIMA, T. \& WAKABAYASHI, K., 1994. Dietary carcinogens and mammary carciongenesis. Cancer, 74:1063-1069.

OBERG, K., 1990. A economia Terena no Chaco. Terra Indígena, 7:20-40.

OLIVEIRA, R. C., 1976. Do Índio ao Bugre: O Processo de Assi milação dos Terena. 2a Ed. Rio de Janeiro: Francisco Alves.

PARKIN, D. M.; MUIR, C. S.; WHELAN S. L.; GAO, Y. T.; FERLAY, J. \& POWELL, J., 1992. Cancer Incidence in Five Continents. IARC Scientific Publications 120. Oxford: Oxford University Press/International Agency for Research on Cancer.

PAWLEGA, J., 1992. Breast cancer and smoking, vodka drinking and dietary habits. A case-control study. Acta Oncologica, 31:387-392.

ROSE, D. P., 1990. Dietary fiber and breast cancer. Nutrition and Cancer, 13:1-8.

SMITH, S. J.; DEACON, J. M. \& CHILVERS, C. E., 1994. Alcohol, smoking, passive smoking and caffeine in relation to breast cancer risk in young women. UK National case-control study group. British Journal of Cancer, 70:112-119.

SWANSON, C. A.; COATES, R. J.; SCHOENBERG, J. B.; MALONE, K. E.; GAM MON, M. D.; STANFORD, J. L.; SHORR, I. J.; POTISCHMANNA, L. A. \& BRINTON, L. A., 1996. Body size and breast cancer risk among women under age 45 years. American Journal of Epidemiology, 143:698-706.

TAVANI, A.; GALLUS, S.; LA VECCHIA, C.; NEGRI, E.; MONTELLA, M.; DAL MASO, L. \& FRANCESCHI, S., 1999. Risk factors for breast cancer in women under 40 years. European Journal of Cancer, 35: 1361-1367.

YUAN, J. M.; WANG, Q. S.; ROSS, R. K. \& HENDERSON, B. E. \&YU, M. C., 1995. Diet and breast cancer in Shangai and Tianjin, China. British Journal of Cancer, 71:1353-1358.

ZIEGLER, R. G., 1997. Anthropometry and breast cancer. Journal of Nutrition, 127(Sup.):924S-928S. 